(Aus dem Institut für allgemeine und experimentelle Pathologie in Wien.)

\title{
Reizversuche am Nucleus caudatus des Hundes.
}

Von

Dr. Arthur Sehüler.

(Hierzu Tafel XXI.)

Ueber die Function des Schweifkernes wissen wir auch heutzutage sehr wenig Sicheres. In einer vor Kurzem erschienenen Arbeit ${ }^{1}$ ) habe ich eine Zusammenstellung der in der Literatur enthaltenen Angaben über die Folgen der experimentellen SchweifkernZerstörung gegeben und dabei hervorgehoben, dass die Erscheinungen, welche bisher auf Grund der Exstirpations versuche dem nucleus caudatus zugeschrieben wurden, zum grössten Theil auf Nebenverletzungen $\mathrm{zu}$ beziehen seien. Ich habe in dieser Arbeit eine Methode zur möglichst isolirten Schweifkern-Zerstörung beim Hunde beschrieben und zwei nach dieser Methode operirte Fälle, wo nach genauer klinischer Beobachtung die anatomische Untersuchung auf Serienschnitten vorgenommen werden konnte, mitgetheilt. Ich konnte kein Symptom feststellen, welches mit Sicherheit auf die Läsion des Nucleus caudatus hätte bezogen werden können.

Eine zweite Methode, die Methode der Reiz versuche, welche, wenn sie auch keineswegs so eindeutige Ergebnisse liefern kann wie gelungene Exstirpationsversuche, doch gerade oft geeignet ist, rasch den richtigen Weg $\mathrm{zu}$ weisen, ist weit häufiger am Nucleus caudatus ausgeführt worden als die Exstirpationsmethode. Um so auffallender ist es, dass auch damit bisher so wenig sichere Ergebnisse zu Tage gefördert wurden.

Dass bei elektrischer Reizung des Nucleus caudatus eine ganze Fülle von Effecten zu beobachten ist, konnte schon zu der Zeit, als

1) Schüller, A., Experimente am Nucleus caudatus des Hundes. Jahrbücher für Psychiatrie und Neurologie Bd. 22. 1902.

E. Pflüger, Archiv für Physiologie. Bd. 91. 
man die Reizungsversuche an der Hirnrinde zu üben begann, nicht verborgen bleiben. Allein schon die ersten Beobachter konnten bezüglich der Deutung der vom Streifenhuggel erzielten Reizerscheinungen zu keinem befriedigenden Ergebnisse gelangen, da sie stets mit der Theilnahme der Kapsel an den beobachteten Effecten zu rechnen hatten. Man bemühte sich auf verschiedene Weise, diesen störenden Factor auszuschalten; das Resultat dieser Bemühungen war, dass der Nucleus caudatus von einem Theil der Beobachter, welcher bezüglich der motorischen Effecte zu negativen Resultaten gelangte, als absolut unerregbar bezeichnet wurde, während andere ibn ebenso entschieden als erregbar bezeichneten.

Ich will daher versuchen, auf Grund einer grösseren Zahl eigener Versuche die folgenden zwei Fragen zu beleuchten: 1. W elche Erscheinungen bieten sich bei der Reizung des Schweifkernes dar? 2. Inwieweit sind diese Reizerscheinungen für die Klarstellung der functionellen Bedeutung des Nucleus caudatus zu verwerthen? Vorweg möchte ich bemerken, dass ich bei meinen Versuchen nicht alle Phänomene analysiren konnte, mich vielmehr auf die Beobachtung der Erscheinungen von Seiten der Skeletmuskulatur, der Athmung, der Circulation und der Harnblase beschränkt habe und in meinen bisherigen Experimenten den Veränderungen von Seiten der Iris und des Darmtractes, welche gelegentlich beschrieben wurden, keine Aufmerksamkeit gesehenkt habe.

Die Methode, welche ich bei den elektrischen Reizversuchen angewendet habe, ist kurz folgende: Der Hund wird mit Aether narkotisirt, das Schädeldach in grosser Ausdehnung entfernt, die Falx mit den sinus longitudinales vorne und hinten mittelst je zweier Pincetten abgeklemmt und hierauf vorn und hinten zwischen den Klemmen durchschnitten: das abgetrennte Stück der Falx wird entfernt; hierauf wird der Spalt zwischen den beiden Hemisphären mit Wattetampons allmählich erweitert, bis schliesslich in der Tiefe der Balken sichtbar ist; dieser wird sagittal durchtrennt, derart, dass der Seitenventrikel eröffnet und in demselben der Nucleus caudatus sichtbar wird. (Die eben beschriebene Methode ist von Lo Monaco (16) zur Blosslegung der subcorticalen Ganglien angegeben worden.) Die elektrische Reizung wird mittelst zweier geknöpfter Platinelektroden ausgeführt; als Stromquelle dient ein Schlittenapparat mit Chromsäureelement. 
Ich will zunächst die Reizeffecte, welche an der Skeletmusk u latur zu beobachten sind, besprechen.

Die ältere Literatur dieses Gegenstandes ist von $\mathrm{Ziehen} \mathrm{(34)}$ (1890) zusammengestellt worden. Longet fand das Striatum völlig unempfindlich gegen mechanische Reizung. Nothnagel, der mit Injectionen von Chromsäure an Kaninchen experimentirte, beschrieb als Folge der Reizung: Deviation der Beine, Verkrümmung der Wirbelsäule (concav nach der Seite der Läsion und zuweilen auch Kyphose). Reizung des Nucleus caudatus an einer bestimmten Stelle des freien Randes bewirkte nach 2-10 Minuten ein Umherhüpfen des Thieres, das schliesslich in ein wildes Umhersturzen, zum Theil mit Reitbahnbewegungen und öfterem Anprall an die Wand, überging (Nodus cursorius). Burdon-Sanderson beobachtete bei faradischer Reizung des Streifenhügels der Katze nur Bewegungen der gekreuzten Muskulatur, diese aber auffälliger Weise schon bei schwächeren Strömen als bei Reizungen der Hirnrinde. Carville und Duret schreiben dem Nucleus caudatus eine gewisse Erregbarkeit zu. Glicky und Eckhard, desgleichen Braun und Solt mann fanden den Streifenhügel durchaus unerregbar für elektrische Reize. Franck-Pitres constatirten Unerregbarkeit sowohl der freien Oberfläche als der Schnittfläche des Nucleus caudatus.

Sehr wichtig ist die Arbeit von Minor (20): „Ueber die Bedeutung des Corpus striatum (1882)." Am meisten Interesse bietet deren zweiter Abschnitt, welcher die elektrische Erregbarkeit des Corpus striatum (hauptsächlich des Nucleus caudatus) behandelt. Bei Reizung der blossgelegten Oberfläche des Nucleus caudatus erhielt Min or Bewegungen der contralateralen Extremitäten nebst Pleurothotonus. Doch ein Strom von derselben Stärke brachte auch immer von der benachbarten Gegend des Stabkranzes aus Bewegungen hervor. Bei geringer Stromstärke konnten von der Oberfläche des Nucleus caudatus aus keine Bewegungen mehr erzielt werden, wohl aber von der Kapsel. Es gelang ihm schliesslich folgender Versuch: Er entfernte einem Hunde die ganze motorische Zone einer Hemisphäre, und nach Abwartung der Ausbildung der secundären Pyramidenbahndegeneration, welche später durch mikroskopische Untersuchung festgestellt wurde, unternahm er die Elektrisation des Nucleus caudatus der operirten Seite. Es zeigte sich, dass Reizung an der Oberfläche dieses Ganglions mit einem Strom von ausserordentlicher Kraft gar keine Bewegung mehr hervorrufe. 
Joh annsen konnte vom Nucleus caudatus typische Epilepsieinsulte nur mit solchen Stromstärken hervorrufen, dass er einen Uebergang von Stromschleifen auf die seiner Anschaung nach epileptogene Zone (Rinde und Linsenkern) annahm.

Ferrier blieb dabei, dass Reizung der ventricularen Fläche des Nucleus caudatus zu allgemeiner Muskelcontraction der gegenüberliegenden Seite mit Pleurothotonus führt.

Ziehen (33 und 34) constatirte, dass beim Kaninchen mechanische Reizung des Nucleus caudatus ohne Effect bleibt. Ein Nodus cursorius für mechanische Reizung existirt nicht. Faradische Reizung löst Mastication, Flimmern der Lippen, Drehungen des Kopfes nach der gekreuzten Seite, tonische Contractionen der gekreuzten und in schwächerem Grade der gleichseitigen Beine aus. Bei längerer Reizdauer entwickelt sich ein wohl charakterisirter tonischer Krampfanfall. Ziehen führte diese Erscheinungen bei faradischer Reizung zum grössten Theil auf Stromschleifen in die innere Kapsel zurück.

Wieting (32) stellte die Beziehungen des Nucleus caudatus zum epileptischen Anfall in Abrede.

Aus der letzten Zeit stammt eine Arbeit von Prus (25) über Reizversuche am Nucleus caudatus, deren Ergebnisse zu denen der Mehrzahl früherer Autoren mehrfach in Widerspruch stehen. Seine Untersuchungen sind an Hunden gemacht. Die Reizung wurde, meist ohne Narkose, nach Abkappung der Hemisphären mit Hülfe des elektrischen Stromes vorgenommen. Es ergab sich, dass im Allgemeinen gewisse Bewegungserscheinungen sowie Sistirung der Respiration, Steigerung des Blutdruckes und Pulsverlangsamung auftreten. Was die Bewegungserscheinungen betrift, so konnte Prus, je nach dem Orte der Reizung, tonische oder clonische Krämpfe oder aber den Laufbewegungen ähnliche Bewegungen oder endlich typische Epilepsieanfälle hervorrufen. Tonische Krämpfe traten bei Reizung der hinteren und äusseren Partien des Streifenhügels auf, klunische Krämpfe bei Reizung der mittleren Partien. Den Laufbewegungen ähnliche Bewegungen machte das Versuchsthier, sobald der vordere $\Lambda$ bschnitt des Streifenhügels gereizt wurde. (Bei allen diesen Bewegungsformen erkannte Prus dann noch Unterschiede je nach der. Stärke des angewendeten Reizes.) Ein Epilepsieanfall wurde am ehesten nach Reizung des medialen oder vorderen Abschnittes des Streifenhügels erhalten. Derselbe begann gewöhnlich 
mit fibrillärem Zittern der Schnauzenmuskulatur; dann traten tonische Kränpfe in der Muskulatur des Thorax und der Extremitäten, zuletzt klonische Anfälle auf. Um festzustellen, ob die motorischen Reizerscheinungen nicht reflectorisch, das heisst auf Reizung sensibler Fasern hin, zu Stande kommen, machte Prus eine Anzahl von Versuchen, in welchen er den Nucleus caudatus mit $10 \%$ iger Cocaïnlösung anästhesirte. Er fand auch jetzt noch, je nach dem Orte der Reizung, entweder nur tonische oder klonische Krämpfe; dagegen war es nicht mehr möglich, Laufbewegungen und Epilepsieanfälle auszulösen. Diese sind also Reflexerscheinungen. Um den Uebergang von Stromschleifen auf die innere Kapsel auszuschalten, experimentirte Prus folgendermaassen: Er durchschnitt zuvor beide Pyramiden und anästhesirte den Nucleus caudatus mit Cocain. Es traten auch jetzt, bei Reizung des anästhesirten Nucleus candatus, tonische bezw. klonische Krämpfe an beiden Körperhälften auf. Prus schloss daraus: Es kann keinem Zweifel unterliegen, dass ton ische bezw. klonische Krämpfe nicht durch Reizung der Pyramidenbahn in der Coronaradiata entstehen, sondern von der Reizung der in den Streifenhügeln befindlichen Zellen und Nerven abhängig sind. Auf welcher Bahn diese Reize gehen, ist, wie Prus selbst sagte, schwer zu entscheiden. Er nimmt für diesen Zweck eine motorische Bahn in Anspruch, welche den hinteren Abschnitt des Streifenhügels mit dem vorderen Vierhügelpaar, und eine andere motorisehe Bahn, welche den mittleren Abschnitt des Streifenhügels mit dem hinteren Vierhügelpaar verbindet. -

Ich habe, um den Einfluss des Nucleus caudatus auf die Bewegungen der Skeletmuskulatur festzustellen, die folgenden Versuche ausgeführt.

\section{Versuch A am 27. Juli 1901.}

Freilegung des Nucleus caudatus beider Seiten nach der Methode von Lo Monaco. Reizung der ventricularen Oberflächedes Schweifkerns bei Rollenabstand $80 \mathrm{~mm}$. Bei Anlegen der Elektroden ganz vorne am Kopfe des linken Nucleus caudatus ist kein Effect zu erzielen. Gleich dahinter findet sich eine Stelle für die obere Extremität; es tritt Streckung des Vorderfusses der Gegenseite und Bewegung der Schulter nach aussen auf. Medialwärts von dieser Stelle findet sich eine Stelle für den Nacken. Vom hinteren Antheil des Schweifkernes lassen sich Bewegungen der hinteren Extremität auslösen und zwar Anziehen des Beines durch Beugung in Hüfte und Knie. Noch weiter hinten éine Stelle für Zukneifen des Auges. Am Nucleus caudatus der rechten 
Seite lässt sich vom vorderen Antheil aus noch Bewegung der linken Ohrmuschel nach hinten auslösen. Bemerkenswerth ist, dass alle Reizstellen, mit Ausnahme der für den Nacken, im lateralen Schweifkernantheil liegen.

Bei stärkeren Strömen treten tetanische Contractionen der Gesammtmuskulatur der Gegenseite auf.

\section{Versuch B am 29. Juli 1901.}

Freilegung beider Schweifkerne nach der Methode ron Lo Monaco. Reizung der ventricularen Oberfläche. Vom linken Nucleus caudatus: entsprechend der Mitte desselben, mehr basalwärts, Zuckungen am Halse bei Rollenabstand $70 \mathrm{~mm}$. Weiter hinten und lateral bei $85 \mathrm{~mm}$ Rollenabstand) starkes Anziehen der rechten hinteren Extremität. Noch weiter hinten Zuckungen an den Lidern (bei Rollenabstand $100 \mathrm{~mm}$ ). -

Vom rechten Nucleus caudatus, und zwar von dessen vorderen Antheil, Contractionen am Halse (bei $90 \mathrm{~mm}$ Rollenabstand).

Es wird hierauf durch Abkappen der Hemisphäre der Querschnitt der Capsula interna freigelegt und gereizt. Man erhält isolirte Zuckungen der vorderen, der hinteren Extremitäten und des Gesichtes bei Rollenabstand $140 \mathrm{~mm}$.

In diesen beiden ersten Versuchen konnte also festgestellt werden, dass durch elektrische Reizung verschiedener Punkte auf der Oberfläche des Nucleus caudatus isolirte Zuckungen der verschiedenen Körpertheile der Gegenseite hervorgerufen werden können. Durch Combination der Ergebnisse beider Versuche ergibt sich die folgende Topographie der Reizpunkteauf der Schweifkernoberfläche, angeführt in der Richtung von vorn nach hinten:

1. Schnauze und Ohr;

2. vordere Extremität;

3. hintere Extremität;

4. Lider;

5. medialwärts von der Stelle für die obere Extremität die Stelle für die Nackenmuskulatur.

Es zeigte sich dabei, dass die Reizstellen alle, mit Ausnahme der für den Nacken, weit lateralwärts, also in unmittelbarer Nachbarschaft der Kapsel lagen; ferner, dass von dem Kapselquerschnitt selbst schon bei geringeren Stromstärken isolirte Zuckungen ausgelöst werden konnten. -

Um nun correspondirende Stellen des Nucleus caudatus und der Capsula interna bezüglich der Leichtigkeit der Auslösung von Zuckungen mit einander zu vergleichen, wurde eine Reihe von Versuchen ausgeführt, in welchen (an der Oberfläche von Schnitten) benachbarte Schweifkern- und Kapselstellen in unmittelbarer 
Aufeinanderfolge gereizt wurden. In Versuch $\mathrm{D}$ wurde dieser Vergleich an Frontalschnitten, in den Versuchen $\mathrm{C}, \mathrm{E}, \mathrm{F}$ und $\mathrm{G}$ an Horizontalschnitten angestellt.

\section{Versuch $C$ am 13. Angust 1901.}

Freilegung des Schweifkernes der linken Seite; hernach Horizontalsehnitt durch die linke Hemisphäre im Niveau des dorsalen Schweifkernpoles. Von der Kapsel aus konnten bei Rollenabstand $120 \mathrm{~mm}$ isolirte Zuckungen des Vorderbeines, der Obren und Schnauze von den entsprechenden Reizstellen aus erhalten werden; gleich darauf ergab Reizung des Schweifkernes erst bei $80 \mathrm{~mm}$ Rollenabstand Zuckungen des Hinterbeines (vom hinteren Antheil aus) und des Vorderbeines (vom vorderen Antheil aus).

Auf der rechten Seite erfolgten von der Kapsel aus Zuckungen bei Rollenabstand 120--80 mm, vom Schweifkern aus bei $90--70 \mathrm{~mm}$.

Versuch D am 15. August 1901.

Es werden (abwechselnd rechts und links) Frontalschnitte durch die Hemisphäre angelegt, drei an der Zahl; der vor: derste legt den Kopf des Schweifkernes, der mittlere den Körper, der hinterste den Schweif frei. Die Blutung aus der Arteria lenticulo-striata ist eine beträchtliche. Die Reizung der Oberfläche des vordersten Schnittes ergibt: Von der Kapsel aus bei Rollenabstand $100 \mathrm{~mm}$ Zuckung des Hinterbeines, vom Nucleus caudatus Zuckungen erst bei $60 \mathrm{~mm}$. Dabei ist deutlich zu sehen, dass, wenn man vom lateralen, dorsalen Antheil des Schweifkernes allmählich die Elektroden nach Aussen verschiebt, das Hinterbein zu zucken beginnt. Von der Oberfläche des zweiten Schnittes erhält man von der Kapsel aus: Dorsal Zuckungen an Schnauze und Lidern, ventral Zuckung der Hinterextremität; vom Nucleus caudatus keine Effecte. Ebenso. verhält es sich bei Reizung der Oberfläche des dritten Schnittes.

\section{Versuch E am 13. September 1901.}

In diesem Versuche konnten bei Erschöpfung des Thieres von der Kapsel aus noch sehr gut Zuckungen der Schnauze, des Halses, der Extremitäten hervorgerufen werden, vom Schweifkern hingegen wurde selbst bei stärkeren Strömen kein Effect mehr erzielt.

\section{Versuch $\mathbf{F}$ am 28. Januar 1802.}

Blosslegung beider Schweifkerne nach der Methode von Lo Monaco. Reizung der ventrikularen Fläche des rechten Nucleus caudatus, der Mitte desselben entsprechend:

bei Rollenabstand $90 \mathrm{~mm}$ kein Effect;

$80, n, n$

60 \#mporheben des Nackens und des Rumpfes.

Reizung der Mitte der Oberfläche des linken Nucleus caudatus:

bei $80 \mathrm{~mm}$ kein Effect;

$" 75$, Anziehen der Hinterpfote und Rumpfbewegungen. 
Sodann wurde durch Abkappung der Hemisphären die Kapsel beiderseits freigelegt. Durch abwechselnde Reizung von benachbarten Schweifkern- und Kapselstellen konnte nunmehr sehr schön der Unterschied in der Erregbarkeit ziffernmässig festgestellt werden.

Linke Hemisphäre:

\begin{tabular}{c|c|c|c}
\hline $\begin{array}{c}\text { Rollen- } \\
\text { abstand }\end{array}$ & Ort & vom Schweifkern & von der Kapsel \\
\hline $100 \mathrm{~mm}$ & Mitte & kein Effect & $\begin{array}{c}\text { Bewegung des rechten } \\
\text { Hinterbeines. }\end{array}$ \\
\hline $95 \mathrm{~mm}$ & Mitte & kein Effect & $\begin{array}{l}\text { Starke Bewegungen (na- } \\
\text { mentlich Laufbewegungen } \\
\text { des rechten Hinterbeines } \\
\text { und Rumpf bewegungen) }\end{array}$ \\
\hline $90 \mathrm{~mm}$ & Mitte & kein Effect & $\begin{array}{c}\text { Deutliche Bewegungen der } \\
\text { Hinterpfote }\end{array}$ \\
\hline $85 \mathrm{~mm}$ & Mitte & $\begin{array}{l}\text { Deutliche Rumpfbewe- } \\
\text { gung, geringe Extremi- } \\
\text { tätenzuckungen }\end{array}$ & $\begin{array}{c}\text { Deutliche Extremitäten- } \\
\text { bewegungen }\end{array}$ \\
\hline
\end{tabular}

Rechte Hemisphäre:

\begin{tabular}{c|c|c|c}
\hline $\begin{array}{c}\text { Rollen- } \\
\text { abstand }\end{array}$ & Ort & vom Schweifkern & von der Kapsel \\
\hline $100 \mathrm{~mm}$ & Mitte & kein Effect & Extremitätenbewegungen \\
\hline $95 \mathrm{~mm}$ & Mitte & kein Effect & \\
\hline $90 \mathrm{~mm}$ & Mitte & $\begin{array}{c}\text { Leichte Bewegung der } \\
\text { linken Hinterpfote, ge- } \\
\text { ringe Rumpfbewegung }\end{array}$ & $\begin{array}{c}\text { Extremitäten- und Rumpf- } \\
\text { bewegungen }\end{array}$ \\
\hline
\end{tabular}

Es wird nummehr auf der linken Seite der Schweifkern durch einen Sagittalsehnitt von der benachbarten Kapsel abgetrennt und ein dünner Wattestreifen in den Spalt eingelegt. Man erhält jetzt bei Rollenabstand $50 \mathrm{~mm}$ rom Schweifkem keinen Effect, von der Kapsel tetanische Contractionen.

\section{Versuch $G$ am 31. Januar 1909.}

Blosslegung des Schweifkernes der rechten Seite und Horizontalschnitt durch die innere Kapsel. Bei Rollenabstand $100 \mathrm{~mm}$ von der Kapsel aus Zuckungen im Gesicht der Gegenseite, am Nacken, an der hinteren und theilweise auch der vorderen Extremität; vom Schweifkern $\because$. Von diesem aus erst bei $70 \mathrm{~mm}$ Rollenabstand Zuckungen der hinteren Extremität (vom hintersten Antheil aus), beider Extremitäten (von der Mitte aus), des Nackens (vom vorderen Antheil aus). - 
Die eben im kurzen Protokollauszuge mitgetheilten Versuche A-G zeigten, dass bei elektrischer Reizung der Schweifkernoberfläche deutliche Effecte von Seiten der Skeletmuskulatur auszulösen sind. Die Bewegungen der Extremitäten bestanden zumeist in einfachen tonischen Anspannungen, seltener in combinirten Bewegungen im Sinne von Laufbewegungen. Die Versuche zeigten ferner, dass eine Differenzirung der Reizstellen für die verschiedenen Körperregionen auf der Schweifkernoberfäche besteht. Da jedoch einerseits die am Schweifkern aufgefundene Topographie der Reizpunkte mit der für die innere Kapsel (beim Hunde namentlich von Franck-Pitres) festgestellten übereinstimmt, andererseits die vom Schweifkern erhaltenen Effecte stets schon bei geringeren Stromstärken von den benachbarten Kapselstellen aus zu erzielen waren, lag die Annahme nahe, dass die beobachteten Körperbewegungen durch die Reizung der von Stromschleifen getroffenen Fasern der Capsula interna veranlasst waren.

Zur Sicherung dieser Aunahme wiederholte ich den oben erwähnten M in or'schen Versuch; ich achtete jedoch dabei nicht bloss auf die Erscheinungen von Seiten der Skeletmuskulatur, sondern auch auf die von Seiten der Respiration, der Circulation und der Harnblase auftretenden Veränderungen. Ueber diese letzteren werde ich weiter unten an entsprechender Stelle Mittheilung machen.

\section{Versuch H.}

Männlicher Bastard-Sehäferhund. Demselben wurde am 5. Febrnar 1902 in Morphium-Aethernarkose die rechtsseitige Fxtremitätenregion in der von Munk angegebenen Ausdehnung exstirpirt und die Markmasse des Centrum semiovale circulär um den Defect umschnitten. Das Thier, welches bereits asn nüchsten Tage herumlicf und in der Folgezeit normalen Wundverlanf sowie keinerlei Störung des Allgemeinbefiudens zeigte, wurde drei Wochen lang genau beobachtet und die bekannten Symptome der linksseitigen Hemiparese an ihm constatiert. Am 25. Februa 1902 wurde der Reizversuch gemacht. In Aethernarkose wurde der Nucleus caudatus beider seiten freigelegt und gereizt. Es zeigte sich, dass vom Nucleus caudatus der linken seite bei mittelstarken Strömen deutliche Körperbewegungen hervorgerufen werden konnten, während vom Nucleus candatus der rechten (operirten) Seite keine Bewegungen errielt wurden, obensowenig von der benachbarten Kapsel, selbst bei vollständig übereinandergeschobenen Rollen. Die anatomische Untersuchung des betreftenden Gchirnes an Marchi-Schnitten in der Gegend des Hirnschenkels zeigte ausgedehnte Degeneration des rechten pes pedunculi. 
Das Ergebniss dieses Versuches, zusammengehalten mit den Resultaten der übrigen Versuche, sichert also die oben ausgesprochene Vermuthung, dass die vom Schweifkern aus erzielten Körperbewegungen auf die Reizung der benachbarten Kapselfasern zurückzuführen seien.

Einer Gruppe der Körperbewegungen hahe ich eine besondere Versuchsreihe gewidmet, den Respirationsbewegungen. Bevor ich die bei Reizung des Nucleus caurlatus erhaltenen Veränderungen der Respiration bespreche, will ich die bezüglichen literaturangaben kurz zusammenstellen. Danilewsky (10) fand bei seinen Experimenten an jungen Hunden und Katzen, dass nur die Reizung der Cauda corporis striati und der nächstliegenden Theile die Respiration charakteristisch veränderte. Diese Veränderung, welche schnell erfolgt, besteht in einer Verlangsamung mit anfänglich tiefer Respiration. Bei mittelstarker Reizung erscheint der Effect in Form einer tiefen Inspiration mit darauf folgender langsamer Exspiration und schliesslich vollständigem Athmungsstillstand, welch' letzterer nicht selten die Reizung überdauert. Danile w sky schliesst, dass durch Reizung des Corpus striatum (vielleicht nur des N. Lentiformis) eine Veränderung der Respiration hervorgerufen werden kann, welche der psychoreflectorischen, die Gemüthsbewegungen begleitenden, ähnlich ist. Schukovsky (27) konnte bei Hunden durch Reizung der grauen Substanz im vorderen Abschnitt des Sehhügels und der Cauda corporis striati Anhalten der Athembewegungen erzielen. Prus (25) sagt: Der Einfluss der Reizung des Nucleus caudatus auf die Respiration gibt sich in der Regel in der Sistirung der Athmung im Momente des Inspiriums kund, zumal bei Reizung der hinteren Partien. Bei Reizung des mittleren Abschnittes hört die Respiration für eine gewisse Zeit ebenfalls auf. Später aber erscheint eine leichte Beschleunigung der Respiration. Wird der vordere Abschnitt des Streifenhügels gereizt, dann dauert die Sistirung der Athmung nur kurze Zeit; hernach wird die Respiration gewöhnlich beschleunigt. Nach Cocaïnisirung des Streifenhügels war es nicht mehr möglich, die Sistirung der Respiration auszulösen. -

Ich habe die Athmungsversuche in der Art angestellt, dass ich nach Freilegung des Nucleus caudatus in der Aethernarkose die Athmung mittelst eines an die untere Brustapertur angeschnallten 
Ballons registrirte. An den dahei erhaltenen Curven konnten sehr schön Veränderungen im Rhythmus, ferner auch Verschiebungen des Athmungsniveaus und Veränderungen in der Tiefe der Excursionen erkannt werden.

\section{Yersuch E am 13. November 1901.}

Abkappung der Hemisphären; Reizung der Schweifkerne. Es trat Vermehrung der Athemfrequenz während der Reizdauer auf; beispielsweise: vor der Reizung 26 Athemzüge per Minute, während der Reizung 30, hernach wieder 26. - Bei einer nächsten Reizung Beschleunigung von 26 auf 33.

\section{Versuch F am 28. Januar 1902.}

In diesem Versuch wurde zuerst der Nucleus caudatus rechts und links, meist in der Mitte seiner ventricularen Fläche, gereizt; hernach wurde durch Horizontalschnitt die Kapsel freigelegt und nun abwechselnd Schweifkern und correspondirende Kapselstelle gereizt.

\begin{tabular}{|c|c|c|c|c|c|}
\hline \multirow{3}{*}{ Reizstelle } & \multirow{3}{*}{$\begin{array}{l}\text { Strom- } \\
\text { stärke }\end{array}$} & \multicolumn{3}{|c|}{$\begin{array}{c}\text { Frequenz der Athmung } \\
\text { per Minute }\end{array}$} & \multirow{3}{*}{$\begin{array}{l}\text { Athmungs- } \\
\text { niveau }\end{array}$} \\
\hline & & vor & während & nach & \\
\hline & & \multicolumn{3}{|c|}{ der Reizung } & \\
\hline $\begin{array}{l}\text { 1. Schweifkern Mitte } \\
\text { rechts }\end{array}$ & $90-70$ & - & $\begin{array}{l}\text { Keine Ver- } \\
\text { änderung }\end{array}$ & - & - \\
\hline $\begin{array}{l}\text { 2. Sehweifkern Mitte } \\
\text { rechts }\end{array}$ & 60 & 25 & 40 & 32 & - \\
\hline $\begin{array}{l}\text { 3. Schweifkern Mitte } \\
\text { rechts }\end{array}$ & 60 & 29 & 57 & 30 & Senkung \\
\hline $\begin{array}{l}\text { 4. Schwoifkern Mitte } \\
\text { rechts }\end{array}$ & 60 & 22 & 32 & 26 & - \\
\hline $\begin{array}{l}\text { 5. Schweifkern Mitte } \\
\text { links }\end{array}$ & 70 & 14 & 30 & 20 & - \\
\hline $\begin{array}{l}\text { 6. Schweifkern Mitte } \\
\text { links }\end{array}$ & 80 & 24 & 28 & 25 & - \\
\hline $\begin{array}{l}\text { 7. Schweifkern Mitte } \\
\text { links }\end{array}$ & 75 & 23 & 98 & 30 & - \\
\hline Schweifkern links & 100 & 16 & 21 & 18 & - \\
\hline Kapsel links & 100 & 12 & 25 & 16 & - \\
\hline Schweifkern links & 90 & 14 & 35 & 16 & - \\
\hline Kapsel links & 90 & 15 & 10 & 16 & Unregelmässig \\
\hline Schweifkern links & 95 & 15 & 18 & 17 & - \\
\hline$\overline{\text { Kapsel links }}$ & 95 & 14 & 17 & 18 & - \\
\hline
\end{tabular}




\begin{tabular}{|c|c|c|c|c|c|c|}
\hline \multirow{3}{*}{\multicolumn{2}{|c|}{ Reizstelle }} & \multirow{3}{*}{$\begin{array}{l}\text { Strom- } \\
\text { stärke }\end{array}$} & \multicolumn{3}{|c|}{$\begin{array}{c}\text { Frequenz der Athmuug } \\
\text { per Minute }\end{array}$} & \multirow{3}{*}{$\begin{array}{l}\text { Athmungs- } \\
\text { niveau }\end{array}$} \\
\hline & & & vor & während & nach & \\
\hline & & & \multicolumn{3}{|c|}{ der Reizung } & \\
\hline \multirow{2}{*}{11.} & Schweifkern links & 90 & 13 & 19 & 17 & - \\
\hline & Kapsel links & 90 & 16 & 24 & 18 & Senkung \\
\hline \multirow{2}{*}{12.} & $\begin{array}{c}\text { Schweifkern links } \\
\text { hinten }\end{array}$ & 85 & 18 & 42 & 26 & - \\
\hline & Kapsel links & 85 & 21 & 22 & 26 & $\begin{array}{l}\text { Abflachung } \mathrm{d} \text {. } \\
\text { Excursionen }\end{array}$ \\
\hline & Schweifkern rechts & 100 & 17 & 17 & 18 & - \\
\hline & Kapsel rechts & 100 & 17 & 46 & 17 & - \\
\hline & Schweifkern rechts & 100 & 16 & 17 & 17 & - \\
\hline & Kapsel reehts & 100 & 16 & 29 & 17 & - \\
\hline 15. & Schweif kern rechts & 95 & 15 & 22 & 17 & - \\
\hline \multirow{2}{*}{16.} & Schweif kern rechts & 90 & 16 & 28 & 18 & - \\
\hline & Kapsel rechts & 90 & 12 & 46 & 15 & - \\
\hline
\end{tabular}

Dieser Versuch zeigte, dass vom Schweif kern aus jedesmal, wenn eine Veränderung gesetzt wurde, eine Vermehrung der Athmungsfrequenz zu Stande kam. Das Niveau der Athmung und die Grösse der Excursionen veränderten sich während der Reizung nicht wesentlich.

Von der benachbarten Kapsel aus wurde einmal Verlangsamung, sonst Beschleunigung der Athmung erhalten; dabei änderte sich zumeist das Niveau der Athmung (im Sinne eines dauernden Inspirationstonus), die Excursionen wurden häufig unregelmässig. Bei schwächeren Strömen war die von der Kapsel aus erhaltene Beschleunigung grösser als die vom Nucleus caudatus; bei stärkeren Strömen änderte sich dieses Verhältniss zu Gunsten des Schweifkernes.

Versuch J am 29. November 1901.

Freilegung des Schweifkernes und der Kapsel der rechten Seite.

\begin{tabular}{|c|c|c|c|c|c|}
\hline \multirow{3}{*}{ Reizstelle } & \multirow{3}{*}{$\begin{array}{l}\text { Strom- } \\
\text { stärke }\end{array}$} & \multicolumn{3}{|c|}{$\begin{array}{c}\text { Frequenz der Athmung } \\
\text { per Minute }\end{array}$} & \multirow{3}{*}{$\begin{array}{l}\text { A thmungs- } \\
\text { niveau }\end{array}$} \\
\hline & & vor & während & nach & \\
\hline & & \multicolumn{3}{|c|}{ der Reizung } & \\
\hline Schweif kern & 85 & 28 & 50 & 40 & $\begin{array}{c}\text { Geringer An- } \\
\text { stieg }\end{array}$ \\
\hline 1. Kapsel & 85 & 40 & 50 & 40 & Anstieg \\
\hline
\end{tabular}




\begin{tabular}{|c|c|c|c|c|c|}
\hline \multirow{3}{*}{ Reizstelle } & \multirow{3}{*}{$\begin{array}{l}\text { Strom- } \\
\text { stärke }\end{array}$} & \multicolumn{3}{|c|}{$\begin{array}{c}\text { Frequenz der Athmung } \\
\text { per Minute }\end{array}$} & \multirow{3}{*}{$\begin{array}{l}\text { Athmungs- } \\
\text { niveau }\end{array}$} \\
\hline & & vor & während & nach & \\
\hline & & \multicolumn{3}{|c|}{ der Reizung } & \\
\hline 2. Schweifkern & 85 & 38 & 108 & 48 & $\begin{array}{c}\text { Allmählicher } \\
\text { Anstieg }\end{array}$ \\
\hline Kapsel & 85 & 48 & 60 & - & - \\
\hline Schweif kern & 90 & 36 & 68 & 36 & $\begin{array}{l}\text { Keine Aende- } \\
\text { rung }\end{array}$ \\
\hline Kapsel & 90 & 36 & 44 & 40 & $\begin{array}{c}\text { Greringer } \\
\text { Anstieg, Ath- } \\
\text { mung wird } \\
\text { unregelmässig }\end{array}$ \\
\hline Kapsel & 100 & 40 & 48 & - & $\begin{array}{l}\text { Anstieg, } \\
\text { grössere Ex- } \\
\text { cursionen }\end{array}$ \\
\hline Schweifkern & 100 & 45 & 52 & - & - \\
\hline 5. Kapsel & 120 & \multicolumn{3}{|c|}{ Keine Veränderung } & - \\
\hline \multirow{2}{*}{ 6. Schweifkern } & 90 & 40 & 60 & 40 & - \\
\hline & 90 & 40 & 46 & - & $\begin{array}{l}\text { Geringer An- } \\
\text { sticg, Ver- } \\
\text { tiefung ader } \\
\text { Excursionen }\end{array}$ \\
\hline Schweif kern vorn & 90 & 42 & 55 & 50 & $\begin{array}{c}\text { Keine Ver- } \\
\text { änderung }\end{array}$ \\
\hline 7. Schweifkern hinten & 90 & 50 & 100 & 43 & Erhebung \\
\hline Kapsel & 90 & 40 & 50 & - & $\begin{array}{l}\text { Bedeutender } \\
\text { Anstieg }\end{array}$ \\
\hline \multirow{3}{*}{$\begin{array}{l}\text { Schweifkern vorn } \\
\text { 8. Schweifkern hinten } \\
\text { Kapsel }\end{array}$} & 90 & 42 & 60 & 42 & \multirow{3}{*}{$\begin{array}{l}\text { - } \\
\text { Erhebung } \\
\text { Errhebung; In- } \\
\text { regelmässigkeit }\end{array}$} \\
\hline & 90 & 42 & 64 & 39 & \\
\hline & 90 & 39 & 45 & - & \\
\hline \multirow{2}{*}{$\begin{array}{l}\text { Sehweif kern hinten } \\
\text { 9. } \text { Kapsel }\end{array}$} & 110 & 43 & $\begin{array}{l}\text { KeineVer- } \\
\text { änderung }\end{array}$ & - & - \\
\hline & 110 & 42 & 60 & - & - \\
\hline 10. Schweifkern vorn & 110 & 44 & 50 & 42 & - \\
\hline Kapsel vorn & 110 & 42 & 44 & - & - \\
\hline \multirow{2}{*}{$\begin{array}{l}\text { Schweifkern hinten } \\
\text { Kapsel hinten }\end{array}$} & 90 & 48 & 105 & 54 & Erhebung \\
\hline & 90 & 54 & 75 & 57 & $\begin{array}{l}\text { Deutliche Er- } \\
\text { hebung }\end{array}$ \\
\hline
\end{tabular}


Dieser Versuch zeigte zunächst wieder, dass durch die elektrische Reizung der Schweifkernoberfäche sehr bedeutende Erhöhung der Athmungsfrequenz erzeugt wird. Bei Strömen mittlerer Stärke ist die Beschleunigung vom Schweifkern aus grösser als die von der Kapsel aus; bei schwachen Strömen kehrt sich dieses Verhältniss um. Bei Schweifkernreizung fehlt häufig jede Veränderung des Athmungsniveaus und der Grösse der Excursionen, während bei der Kapselreizung zumeist Inspirationstonus und Veränderungen der Excursionen auftraten. Jene Reizungen, bei denen die Stelle beachtet wurde, an der die Elektroden angelegt wurden, liessen erkennen, dass der Effect vom vorderen und hinteren Antheil des Schweifkernes verschieden ist. Die Beschleunigung, welche durch Reizung der hinteren Partie des Nucleus caudatus resultirt, ist meist bedentend grösser als die von der vorderen. Dagegen ist sie gewöhnlich nicht so rein; es tritt bei Reizung des hinteren Antheils meist Inspirationstonus dazu. (Siche die Curven 1-4.)

\section{Versuch K am 29. November 1901.}

Freilegung beider Schweifkerne und Horizontalschnitt durch beide Hemisphären. Abwechselnde Reizung von Schweifkern und Kapsel, und zwar sowohl an deren vorderen als hinteren Partien. Es zeigte sich, dass vom vorderen Antheil des Schweifkernes und der Kapsel sehr deutliche Beschleunigung ohne Inspirationstonus ausgelöst wurde. Je weiter nach hinten gereizt wurde, desto deutlicher trat Inspirationstonus auf, während die Beschleunigung sich verminderte. Im Verlaufe dieses Versuches trat in Folge der Narkose Asphyxie auf. Als das Thier nach einigen künstlichen Respirationen wieder zu sich kam, war die Athmungsfrequenz sehr beschleunigt, durchschnittlich 80 per Minute. Auch jetzt konnte durch Reizung des Schweifkernes öfters noch eine deutliche Zunahme der Athmungsfrequenz erzielt werden, während von der Kapsel aus, und zwar von deren hinteren Antheil, zumeist Verlangsamung der Athmung und Inspirationstonus ausgelöst wurde.

\section{Versuch H am 25. Februar 1992.}

Es ist dies jener Versuch, wo, wie bereits oben beschrieben, die Reiz ung des Schweifkernes nach vorheriger Rindenexstirpation und secundärerkapseldegeneration vorgenommen wurde. Es wurde zuerst der Schweifkern der linken, dann der Schweifkern der rechten (operirten) Seite freigelegt. Die Reizung wurde an beiden abwechselnd vorgenommen.

\begin{tabular}{c|c|c|c|c}
\hline & Seizstelle & \multicolumn{3}{|c}{ Athmungsfrequenz } \\
\hline & stärke & vor & während & nach \\
\hline 1. Rechter Schweifkern & 90 & 31 & 31 & 31 \\
\hline 2. Rechter Schweifkern & 80 & 31 & 32 & 30
\end{tabular}




\begin{tabular}{c|c|c|c|c}
\hline \multirow{2}{*}{ Reizstelle } & Strom- & \multicolumn{3}{|c}{ Athmungsfrequenz } \\
\cline { 3 - 5 } & stärke & vor & während & nach \\
\hline Rechter Schweifkern & 70 & 30 & 37 & 30 \\
\hline Linker Schweifkern & 70 & 23 & 45 & 30 \\
\hline Linker Schweifkern & 70 & 33 & 42 & 35 \\
\hline Rechter Schweifkern & 70 & 34 & 34 & 33 \\
\hline 5. Rechter Schweifkern & 65 & 33 & 36 & 34 \\
\hline Rechter Schweifkern & 60 & 34 & 37 & 35 \\
\hline Linker Schweifkern & 60 & 33 & 81 & 37
\end{tabular}

Dieser Versuch zeigte also, dass vom Schweifkern der operirten Seite bei mittleren Strömen keine Beschleunigung, bei stärkeren Strömen eine sehr geringe Zunahme der Athmungsfrequenz erzielt wurde. Vom linken Schweifkern aus kam stets eine bedeutende Beschleunigung zu Stande. -

Ein Ueberblick über die bezüglich der Athmung erhaltenen Resultate ergiht: 1. Vom Nucleus caudatus wurde in allen Fällen durch die Reizung eine Beschleunigung der Athmung erzielt. 2. Von der vorderen Partie des Nucleus caudatus wurde dieselbe am reinsten zu Wege gebracht. Von der hinteren Partie des Nucleus caudatus wurde oft eine noch grössere Beschleunigung erhalten, jedoch meist zusammen mit Inspirationstonus. 3. Von der benachbarten $\mathrm{Kapsel}$ aus wurde eine reine Beschleunigung viel schwieriger erzielt; dieselbe war meist combinirt mit Inspirationstonus, vorne weniger als hinten. Wie vom Nucleus caudatus, so war auch von der Kapsel aus die Beschleunigung meist am grössten bei Reizung im hinteren Antheil. 4. Vergleicht man die bei Reizung correspondirender Stellen des Nucleus caudatus und der Kapsel resultirenden Beschleunigungen, so findet man, dass die Frequenzerhöhung vom Nucleus caudatus ans zumeist grösser ist als die von der Kapsel aus. Nur bei sehr schwachen Strömen wurde zuweilen vom Nucleus caudatus aus keine Frequenzveränder ung mehr erhalten, während die Kapselreizung noch die Athmung beschleunigte. 5. Eine Frequenzverminderung wurde vom Nucleus caudatus niemals, von der Kapsel gelegentlich im hinteren Antheile erhalten. 6. Nach Exstirpation der motorischen 
Region und secundärer Kapseldegeneration war vom zugehörigen Nucleus caudatus aus keine Beschleunigung mehr erzielbar.

Um diese Resultate $z u$ deuten, muss ich die Thatsachen, welche namentlich im Laufe der letzten Jahre über die cerebrale Athmungsinnervation bekannt geworden sind, mit zu Hülfe nehmen.

In den subcorticalen Regionen beschrieb zuerst J. Ott ${ }^{1}$ ) ein auf Reizung die Zahl der Athemzüge lebhaft vermehrendes Centrum im Gewebe zwischen Streifen- und Sehhügel. Wird dasselbe zerstört, so hört auch die durch Wärme erzeugte dyspnoetische Athmungsbeschleunigung (Wärmedyspnoe) auf.

In den Sehhügeln, im Boden des dritten Ventrikels, fand Christiani (7 und 8) ferner ein besonderes Inspirationscentrum, dessen directe Reizung inspiratorisch vertiefte und beschleunigte Athemzüge und selbst Stillstand in der Inspiration bewirkt. Nach Exstirpation desselben konnte er ein exspiratorisch wirksames Centrum in der Substanz der vorderen 4-Hügel, nicht weit vom aquaeductus Sylvii nachweisen.

In den hinteren 4-Hügeln fanden Martin und Booker (18 und 19) ein zweites cerebrales Inspirationscentrum, Lewandowsky (15) ein Inspirationshemmungscentrum. Marckwald (17) schrieb den Bahnen und Kernen der hinteren 4-Hügel und des sensibeln Trigeminuskernes nächst den vagi die grösste Bedeutung für die Auslösung regelmässiger rhythmischer Athmung zu.

Die Literaturangaben über die Beziehungen des Corpus striatum zur Respiration habe ich bereits oben zusammengestellt.

Ueber die Athmungscentren in der Hirnrinde liegen mehrere, zum Theil recht ausführliche Mittheilungen vor.

Danilewsky (10) erhielt zwei Serien von Effecten, je nach der Region, welche er reizte: bei schwacher Reizung der grauen Substanz des suprasylvischen Gyrus beobachtete er Verlangsamung der Athmung mit anfangs tiefer Inspiration; bei Reizung gegen die Basis des Gehirns zu erhielt er eine bedeutende Athmungsbeschleunigung.

Lépine (14) und Bochefontaine (6) konnten Vermehrung der Athmungsfrequenz durch Reizung der Hirnrinde erzielen.

Richet (26) fand an verschiedenen Stellen der Hirnrinde Punkte, deren Reizung unmittelbar die Athmung aufhebt.

1) Citirt nach Landois (13). 
Hermann Munk (21 und 22) gelang es als erstem, die Rindenstellen für die Athmung zu localisiren. Er fand an der oberen Fläche des Stirnlappens eine Region, deren Reizung Stillstand der Athmung mit maximaler Inspirationsstellung des Thorax und tetanische Contraction des Zwerchfelles ergibt; manchmal geht dem Inspirationstetanus eine beschleunigte Athmung voraus, wobei unter grösseren Inspirationen und kleineren Exspirationen Thorax und Zwerchfell mehr und mehr der maximalen Inspirationsstellung sich nähern, bis sie schliesslich in dieser verharren. An der unteren Fläche des Stirnlappens fand $\mathrm{Munk}$ eine zweite Stelle, von der aus die Athmung, aber in entgegengesetztem Sinne, beeinflusst werden konnte. Es tritt bei Reizung derselben Tetanus der Bauch(Exspirations-)Muskeln, oder aber es treten ausserordentlich häufige active Exspirationen ein, die nur zeitweise von einer Inspiration unterbrochen werden.

Franço is - Franck (11) fand, dass die Reizung jedes beliebigen Punktes der motorischen Zone gewisse Veränderungen der Athembewegungen im Gefolge habe, während die Reizung anderer, also nicht motorischer Partien der Rinde die Athmung nicht beeinflusst. Die Veränderungen der Athmung seien Aenderungen der Frequenz. Ein Zusammenhang zwischen dem Orte der Reizung und dom Effecte derselben bestehe nicht.

Unvericht (31) beschrieb ein corticales Hemmungscentrum für die Athmung. Er localisirte es lateral vom Orbiculariscentrum. Reizung dieser Stelle bewirkt sofort eine Verlangsanung der Athembewegungen, meist so, dass die Dauer der exspiratorischen Athempause verlängert wird.

Preobraschensky (24) kam ohue Kenntniss der Unverrichtschen Experimente zu dem gleichen Resultate wie dieser. Ausserdem fand er ein hinter dem Exspirationscentrum gelegenes Inspirationscentrum, durch dessen Reizung inspiratorischer Tetanus des Zwerchfelles hervorgerufen wird.

Die Arbeiten der letzten Jahre haben die von Hermann Munk angegebene Localisation der Athmungsstellen im Wesentlichen bestätigt; in erster Linie die Arbeiten Spencer's (28 und 29$)$.

Spencer fand bei seinen Reizungsexperimenten, welche er an Affen, Hunden, Katzen und Kaninchen anstellte, vier verschiedene Resultate, jedes erreichbar von einer umgrenzten Area der Rinde E. Pflüger, Archiv für Physiologie. Bd. 91. 
und von dieser aus entlang einer bestimmten Bahn weiter caudalwärts verfolgbar.

1. Der erste Effect besteht in einer Verminderung der Thätigkeit des Respirationscentrums der medulla oblongata, welche bis zum Athmungsstillstand führen kann. Das Rindenfeld, von dem aus dieses Resultat erhalten wurde, liegt lateral vom Tractus olfactorius. Von hier aus lässt sich der gleiche Effect rückwärts verfolgen entlang einer Bahn, welche durch die Commissura anterior auf die andere Seite übergeht und an der Seite des Infundibulum in den rothen Kern gelangt.

2. Der zweite Effect besteht in der Steigerung der Thätigkeit des Respirationscentrums. Die Respiration kann bedeutend beschleunigt werden; allerdings, was durch die Beschleunigung gewonnen ist, kann theilweise verloren werden durch die Verminderung der Excursionen. Der Rindenbezirk, von welchem aus die grösste Beschleunigung erzielt werden kann, liegt rings um das obere Ende des Sulcus infraorbitalis (bei Hund und Katze) - der Effect der Beschleunigung kann dann nach rückwärts verfolgt werden. längs einer Bahn, welche durch den Nucleus lentiformis, an der lateralen ventralen Partie der Capsula interna vorbei, zum Tegmentum verläuft.

3. Eine andere gesteigerte Function des Respirationscentrums kann erhalten werden in der Form des "Schnüffelns". Dieser Effect kann erzielt werden von der Schleimhaut des oberen Theiles der Nase, von dem Nervus, dem Bulbus und Tractus olfactorius. Er kann rückwärts verfolgt werden zum gyrus uncinatus des Temporosphenoidal-Lappens; weiterhin vom Uncus hinter dem Tractus opticus zum Hirnschenkel.

4. Der vierte Effect, welcher von der Oberfläche des Gehirns erreicht werden kann, ist ein sehr verbreiteter. Es ist derselbe, wie er durch Reizung eines sensorischen Nerven erhalten werden kann. Der Brustkasten nimmt eine überinspiratorische Stellung an in Folge einer tonischen Contraction der auxiliären Inspirationsmuskeln, während die rhythmische Bewegung der gewöhnlichen Athmungsmuskeln fortdauert. Die Reizung des senso-motorischen Feldes und der absteigenden motorischen Bahn erzeugt dieses Resultat.

In neuester Zeit hat Beyermann (5) Experimente über die cerebrale Athmungsinnervation publicirt. Er kam im Wesentlichen zu den gleichen Ergebnissen wie Spencer. Auch er trennt die 
Rindenstelle, deren Reizung Inspirationstonus erzeugt, von derjenigen, welche Athmungsbeschleunigung bewirkt. Während er von der ersteren Rindenstelle aus die centrifugale Bahn bis in den Hirnschenkel verfolgen konnte, gelang es ihm nicht, die Bahn der Beschleunigung weiter als bis zum Nucleus caudatus zu verfolgen. Er liess daher die Frage offen, ob diese Bahn im Schweifkern nicht eine Unterbrechung erfahre. -

An dieser Stelle können, wie ich glaube, die Ergebnisse unserer Untersuchung ergänzend angefügt werden. Die Thatsache, dass vom Nucleus caudatus so constant Beschleunigung zu erzielen war, hätte zunächst zur Annahme verleiten können, dass im Nucleus caudatus selbst ein Centrum für die Athmungsbeschleunigung enthalten sei. Da nach Rindenexstirpation und secundärer Degeneration der absteigenden Kapselfasern der Effect der Athmungsbeschleunigung vom Nucleus caudatus nicht mehr zu erzielen war, müssen wir die obige Annahme fallen lassen, vielmehr jenen beschleunigenden Effect auf Rechnung der Kapsel setzen. Die Thatsache aber, dass vom Nucleus caudatus die Beschleunigung reiner und zumeist in höherem Grade zu erzielen ist als von der Kapsel, führt zur Annahme einer besonders innigen topischen Beziehung des Nucleus caudatus zu jeneu athmungsbeschleunigenden Kapselfasern. Diese dürten vom Stirnhirn aus in unmittelbarer Nachbarsehaft des Nucleus caudatus, an dessen laterale Seite angeschmiegt, rückwärts ziehen.

Da der Effect der Beschleunigung von der hinteren Partie des Nucleus caudatus grösser ist als von der vorderen, so dürfteı jene Kapselfasern in ihrem weiteren Verlauf nach hinten zu einem geschlossenen Bündel an der lateralen Seite des Nucleus caudatusSchweifes convergiren.

Dass von der Kapsel aus der Effect der Beschleunigung erstens nicht so rein und zweitens nicht in so hohem Grade zu erzielen ist wie vom Nucleus caudatus, dürfte sich wohl mit Leichtigkeit daraus erklären lassen, dass einerseits der Horizontalschnitt der Kajsel keinen so günstigen Angriffspunkt für die Reizung bietet wie die breite Oberfläche des Nucleus caudatus, andererseits aber daraus, dass das Nahebeisammenliegen der verschiedenen -- athmungsbeschleunigenden, athmungsverlangsamenden und inspirations-tonus-erzeugenden - Fasern innerhalb der Corona radiata das Zustandekommen reiner Beschleunigung von der Kapsel aus unmöglich macht. 
Einen anatomischen Beleg für den weiteren Verlauf der erwähnten, an der lateralen Seite des Nucleus caudatus verlaufenden Kapselfasern glaube ich Präparaten entnehmen zu können, die von einem Hunde stammen, an welchem ich den Nucleus caudatus der linken Seite total zerstörte, wo aber gleichzeitig die unmittelbar anschliessenden Kapselfasern mit betroffen wurden. Den Hund liess ich drei Monate nach der Operation am Leben. Die Untersuchung der Hirnschnitte nach Weigert ergab, dass sich im Anschluss an das Zugrundegehen der dem Nucleus caudatus benachbarten Kapselfasern eine Atrophie des medialen Thalamus-Kernes ausgebildet hatte. Es dürfte somit dieser Kern die Endstation der von der Rinde herabziehenden, vermuthlich athmungsbeschleunigenden Kapselfasern sein.

Die eben angeführte anatomische Erfahrung stimmt auch mit den physiologischen Thatsachen überein: bekanntlich hat ja gerare an der erwähnten Stelle Christiani sein Inspirationscentrum des dritten Ventrikels localisirt.

Die betreffenden Fasern sind also ein Theil des sogenannten vorderen Stieles des Thalamus, der bekanntlich aus dem Stirnhirn durch den vorderen Theil der inneren Kapsel zwischen Linsenkern und Schweifkeru direct sagittal gegen den Thalamus zieht.

Ueber die Beziehungen des Nucleus caudatus zur Blutcirculation finden sich in der Literatur mehrere Angaben. Danilewsky (10) constatirte, dass Reizung des vorderen Theiles des Corpus striatum fast obne jede Wirkung auf den Blutdruck bleibt, dass hingegen Reizung der Cauda corporis striati und der nächst anliegenden weissen Substanz fast stets eine sehr merkliche Veränderung des Blutdruckes hervorruft: der Blutdruck steigt, der Puls wird langsamer, die Pulswelle grösser. Bei Ermüdung des Gehirns in Folge vorangegangener bedeutender Reizungen sah Danilewsky im Gefolge der Tetanisirung des Corpus striatum und der angrenzenden weissen Substanz zwei Mal eine grosse Beschleunigung der Herzschläge bei unbedeutendem Herabsinken des mittleren Blutdruckes.

Stricker (30) fand bei Reizung des Corpus striatum, namentlich der vorderen Theile, Steigerung des Blutdruckes ebenso wie bei Reizung der motorischen Region. Im Gegensatze zu Danilewsky erzielte er niemals Pulsverlangsamung. In einem Falle bekam er vom Corpus striatum aus (nach einem kurzen Latenz- 
stadium) eine ausgesprochene Depression, hernach erst eine Steigerung des Blutdruckes.

Prus (25) beobachtete Hebung des Blutdruckes bei Reizung des Striatum. Die stärkste Steigerung erreichte er bei Reizung der medialen Partie des Streifenhügels. Typische Pulsverlangsamung beobachtete er, sobald der mediale oder hintere Abschnitt des Streifenhügels gereizt wurde; dabei hatte die Pulsverlangsamung den Typus der sogenannten Vaguspulse. Nach Cocainisirung des Striatum konnte Prus noch Blutdrucksteigerung hervorrufen, dagegen keine Aenderung in der Pulszahl. -

Ich selbst habe bei drei Versuchen (Versuch E, H, K) die Veränlerungen des Blutdruckes nach Sehweifkernreizung am narkotisirten und spontan athmenden Hunde beobachtet. Es zeigte sich, selbst bei Anwendung stärkerer Ströme, nur eine unbedeutende Veränderung, welche fasst stets zusammenfiel mit lebhaften Körper- und Athembewegungen. Die Veränderung des Blutdruckes bestand in einer geringen Senkung desselben. Diese war um so deutlicher, je stärker die durch die Reizung hervorgerufene Athmungsveränderung, insbesondere die tonische Inspiration, hervortrat. Es dürfte somit die erhaltene Blutdrucksenkung die Folge der Athmungsveränderung gewesen sein.

In weiteren drei Versuchen habe ich die Veränderung des B 1 u tdruckes bei Schweifkernreizung an jungen curarisirten Hunden beobachtet.

\section{Versuch $\mathrm{U}$ am 17. Juni 1902.}

Tracheotomie. Curarisirung. Freilegung beider Nuclei caudati. Blutdruckschreibung aus der Arteria femoralis. Der Blutdruck ist in Folge grösseren Blutverlustes bei der Operation sehr niedrig und hebt sich crst nach Kochsalzinfusion.

Bei Reizung des Schweifkernes tritt eine Blutdrucksteigerung geringen Grades auf. Auch vom Querschnitt der Capsula interna wird eine Steigerung erzielt.

\section{Versuch V am 24. Juni 1902.}

Junger IIund. Tracheotomie, Curarisirung. Freilegung beider Schweifkerne. Blutdruckschreibung aus der Arteria femoralis.

Bei diesem Versuche wurde eine grössere Anzahl von Reizungen an der Oberfäche beider Nuclei candati, an cinem Horizontalschnitte durch dic linke Kapsel und einem Schrägschnitte durch die rechte Kapsel vorgenommen.

Sowohl vom Schweifkern als von der Kapsel aus konnte jedes Mal, wenn ein positiver Effect auftrat, eine Steigerung des Blutdruckes erhalten 
werden. Die höchste, bei der ersten Reizung des linken Nucleus caudatus erzielte Steigerung betrug $140 \mathrm{~mm}$ Quecksilber (Anstieg von 64 auf $204 \mathrm{~mm}$ ). Die Reizung danerte 9 Secunden. 2 Secunden nach dem Einsetzen des Reizes begann die Blutdrucksteigerung; sie erreichte ihren Höhepunkt einige Zeit nach dem Aufhören der Reizung. Auf der Höhe der Blutdrucksteigerung trat eine bedeutende Pulsverlangsamung und Vertiefung der Pulswelle anf.

Um einen Anhaltspunkt für die Lage der wirksamsten Stelle $z u$ finden, wurden an der Oberfläche des Nucleus caudatus sechs verschiedene Bezirke abwechselnd gereizt: im dorsalen und ventralen Antheile je eine vordere, mittlere und hintere Stelle; dabei wurde die Höhe der erzielten Blutdrucksteigerung, die Stromstärke und die Latenzzeit des Eintrittes der Steigerung in Rechnung gezogen. Mit Sicherheit gelang es nicht, eine bestimmte Stelle der Schweif kernoberfläche als die wirksamste ausfindig zu machen; die rasche Abnahme des Effectes bei wiederholter Reizung erschwert die vergleichende Untersuchung verschiedener Bezirke. Im Allgemeinen schien es jedoch, dass der beste Effect vom hinteren Antheil des Schweifkernes ausgelöst werden könne.

\section{Versuch $W$ am 30 . Juni 1902.}

Junger Hund. Tracheotomie, Curarisirung. Freilegung beider Nuclei eaudati. Blutdruckschreibung aus der Arteria femoralis. Schreibung der Bewegung dreier Herzabtheilungen.

In diesem Versuche konnte jedes Mal bei Reizung des Schweifkernes (Rollenabstand $60-70 \mathrm{~mm}$ ) eine bedeutende Blutdrucksteigerung erzielt werden. Dieselbe trat gewöhnlich $2-5$ Secunden nach Einsetzen der Reizung auf; sie erreichte bald nach dem Aufhören der Reizung ihren Höhepunkt, um dann allmählich wieder zurückzugehen. Die höchste Steigerung betrug $40 \mathrm{~mm}$ Quecksilber. Bei Vergleich der Wirkung verschiedener Stellen der Schweif kernoberfläche zeigte es sich, dass der hintere Antheil einen höheren Anstieg veranlasste als der vordere. Wurde die Kapsel durch Horizontalschnitt freigelegt und gereizt, so ergab sich ebenfalls eine beträchtliche Blutdrucksteigerung.

Die Schreibung der Bewegungen dreier Herzabtheilungen (des rechten Vorhofes und beider Ventrikel) ergab, dass die Schweifkernreizung ohne Einfluss auf das Herz selbst blieb. Für die Vervollständigung des Versuehes nach dieser Richtung bin ich Herrn Dr. Winterberg zu bestem Dank verpflichtet.

Die Reizung des Nucleus caudatus hatte also in den drei Versuchen, welche an curarisirten Hunden angestellt wurden, regelmässig eine Blutdrucksteigerung im Gefolge. Dass diese Steigerung nicht durch eine Veränderung der Thätigkeit des Herzens zu Stande kommt, zeigte der Versuch $\mathrm{W}$, wo die Bewegungen dreier Herzabtheilungen registrirt wurden. Sie muss also durch eine Con- 
traction der peripheren Gefässe veranlasst sein. Wahrscheinlich, jedoch nicht sicher ist es, dass der beste Effect vom hinteren Antheile des Schweifkernes ausgelöst werden kann. Eine Veränderung des Pulses, Verlangsamung desselben und Vertiefung der Pulswelle, trat nur bei sehr bedeutenden Steigerungen des Blutdruckes auf der Höhe derselben auf.

Von der Kapsel aus konnte ebenso wie vom Schweifkern aus Blutdrucksteigerung erzielt werden.

Nach den bezüglich der Körperbewegungen gemachten Frfahrungen drängt sich auch bezüglich der vom Schweifkern aus erzielten Blutdruckveränderungen die Vermuthung auf, dass dieselben durch Reizung der benachbarten Kapsel hervorgerufen seien. Wir wissen ja, dass die Grosshirnrinde Einfluss nimmt auf die Gefässinnervation (B ochefoutaine, Danilewsky, François-Franck, Bechterew-Mislawsky). Will man also zu einer richtigen $\mathrm{Ab}$ schätzung der vom Schweifkern selbst veranlassten Blutdruckveränderungen gelangen, so ninss man die Kapselwirkung (durch vorausgegangene Rindenexstirpation) ausschalten. Ich hatte bisher nicht Gelegenheit, ein derartiges verwerthbares Experiment auszuführen. Ich habe wohl in dem mehrfach erwähnten Versuche H, wo ich die Schweifkernreizung nach vorausgegangener Rindenexstirpation und Kapseldegeneration vornahm, auch auf die Veränderungen des Blutdruckes geachtet. So lange das Thier nicht curarisirt war, erhielt ich aus den oben angeführten Gründen keinen verwerthbaren Fffect; nach der Curarisirung an Schlusse des Versuchs aber sank der Blutdruck tief herab, derart, dass durch die Reizung des Schweifkernes auch auf der intacten Seite kein Effect erzielt werden konnte.

Was die Beziehungen des Nucleus caudatus zur Innervation der Harnblase betrifft, so liegen diesbezüglich wenig Untersuchungen vor. Valentin ${ }^{1}$ ) fand bei Reizung der Corpora striata (gerade so, wie bei der des Kleinhirns, der Hirnsehenkel, der Thalami optici) Contraction der Hamblase, während die Reizung der Hemisphären nicht in so constanter Weise wirkte. Von klinischer Seite sind insbesondere Hutchinson, Rezek, Czyhlar ̌̀-Marburg (9) für Beziehungen der Harnblase zum Streifenhügel eingetreten.

Ich bin gerade gegenwärtig mit der genaueren experimentellen

1) Citirt nach Landois (13). 
Prüfung dieser Beziehungen beschäftigt, möchte daher vorläufig nur wenige Worte über diesen Gegenstand mittheilen.

Wurde, nachdem der Schweifkern in Aethernarkose freigelegt worden war, die gefüllte Harnblase durch einen Katheter, welcher in das Cavum derselben hineinreichte, mit einem Manometer verbunden, so stieg das Niveau der Curve bald nach Beginn der Reizung des Nucleus caudatus allmählich in die Höhe; der Anstieg überdauerte eine Zeit lang das Ende des Reizes; hernach fiel die Curve wieder auf das frühere Niveau. Derselbe Effect wurde von der benachbarten Kapsel erhalten. Dabei waren stets Bewegungen des Thieres zu constatiren, so zwar, dass die Steigerung des Blasendruckes auch durch diese veranlasst sein konnten.

Wurden die Körperbewegungen mittelst Curare ausgeschaltet, dann trat auf Reizung des Nuclus caudatus eine Veränderung des Niveaus nicht mehr auf, auch nicht, nachdem die Nervi splanchnici durchschnitten worden waren.

In dem einem Falle, wo ich die Reizung des Schweifkernes nach vorheriger rechtsseitiger Rindenexstirpation und secundärer Kapseldegeneration vornahm (Versuch $\mathrm{H}$ ), fand ich keinerlei Steigerung des Blasendruckes bei Reizung des Schweifkernes der rechten (operirten) Seite, dagegen eine sehr deutliche Steigerung mit allgemeinen Körperbewegungen bei Reizung des linken Schweifkernes.

Was den Sphincter vesica e betrifft, so kann ich nach meinen bisherigen Versuchen über dessen Verhalten bei Schweifkernreizung nichts aussagen. Ich bin gerade mit der Ausführung einer entsprechenden Technik zur Registrirung des Blasenverschlusses beschäftigt.

Wenn ich die Ergebnisse der elektrischen Schweif kernreizung überblicke, so zwingt sich mir die Ueberzeugung auf, dass mit Rücksicht darauf, dass die meisten der vom Nucleus caudatus erzielten Effecte verschwanden, wenn die Wirkung der Kapselfasern durch vorhergegangene Degeneration ausgeschlossen war, in jedem Falle, wo man einen Reizeffect vom Nucleus caudatus beobachtet, dieser Effect erst als dem Schweifkern zugehörig verificirt werden müsste dadurch, dass man Vergleichsversuche mit vorausgegangener Rindenabtragung macht.

Andererseits möchte ich nicht versäumen, darauf aufinerksam zu machen, dass die Schweifkernreizung nach Abtragung 
von Rindenpartien und Abwartung der secundären Degeneration eine brauchbare Methode liefern dürte zur Klarstellung der Localisation von motorischen Functionen in der Hirnrinde; die Reizung des Schweifkernes, von dem wir ja mit grösster Wahrscheinlichkeit sagen kömnen, dass ihm selbst keine motorische Erregbarkeit zukommt, ist in diesem Falle der directen Reizung der stehengebliebenen Rinde und der Kapsel vorzuziehen.

Es wäre diese Methode eine Ergänzung zu der jetzt von verschiedenen Autoren, zuerst von Starlinger, später insbesondere von Probst, Rothmann geübten, nämlich: Durchschneidung der corticofugalen Bahnen in der Gegend der Pyramiden und abwärts davon, Abwartung der Degeneration und hierauf Reizung der Rinde zum Zwecke der Constatirung, welche Partien noch reizbar sind.

Im Anschluss an die elektrischen Reizversuche will ich eine Reihe von "Wärmestich"-Experimenten am Nucleus caudatus des Hundes vorführen, Experimente, welche, von den meisten Autoren als mechanische Reizversuche aufgefasst, die Veründerung der Körpertemperatur nach Einstichen in den Schweifkern demonstriren sollen.

Die Literatur dieses Gegenstandes ist recht unfangreich. Bekanntlich existirt ja fast keine Stelle des Centralnervensystems, welche nicht in Beziehung zu Temperaturveränderungen gebracht worden wäre. Seit dem Jahre 1884 aber steht gerade das Corpus striatum als Hauptcentrum der Temperaturerhöhung im Mittelpunkte des Interesses. In diesem Jahre veröffentlichten Richet in Frankreich, Ott in Amerika und Aronsohn-Sachs in Deutschland ihre Mittheilungen über die Beziehungen des Corpus striatum zur Körpertemperatur. Rich et sagte: Das Corpus striatum seheint mehr als die ubbrigen Partien des Gehirns im Stande zu sein, reflectorisch Hyperthermie zu erzeugen. Ott sagt: In der Nachbarsehaft des Corpus striatum sind Centren, welche eine Beziehung zur Körpertemperatur haben. Aronsohn-Sachs localisirten damals als die Ersten die thermogenetisch wirksame Stelle in die mediale Seite des Nucleus caudatus.

In Laufe der seitdem verflossenen 18 Jahre haben einerseits die citirten Autoren selbst Veränderungen ihrer ursprünglichen Mittheilungen vorgenommen, andererseits erfuhren die bezüglichen Fragen von Seiten einer grossen Anzahl anderer Autoren eingehende 
Berücksichtigung. Eine Zusammenstellung der wichtigsten Arbeiten auf diesem Gebiete findet sich in der ausführlichen Darstellung von It o (12): Ueber den Ort der Wärmebildung nach Gehirnstich, 1899. It o selbst kam zu dem Ergebniss, dass das nervöse Hauptcentrum für Steigerung der Wärmebildung in den Corpora striata gelegen ist. Dieses Hauptcentrum liegt in der Mitte des freien Randes vom Corpus striatum. Von bier gehen die nervösen Bahnen durch die Brücke vermuthlich auf das Gebiet des Sympathicus über. Der Stich in das Corpus striatum verursacht Hyperthermie durch nervöse Erregung, nicht durch Aufhebung einer Hemmung. Im Duodenum steigt nach dem Einstich die Temperatur schneller und zu höherem Maximum an als an irgend einer anderen Stelle. Wabrscheinlich erhöht das gereizte Wärmecentrum zunächst die Thätigkeit des Pankreas und vielleicht auch der Duodenaldrüsen.

In einer vor wenigen Monaten erschienenen Publication fasst Aronsohn(1) seine neuesten Ergebnisse über den Zusammenhang der Temperatursteigerung mit den Wärmecentren, als deren wichtigstes er auch jetzt noch das Corpus striatum bezeichnet, in folgenden Worten zusammen: Das Wesen des Fiebers besteht in einer krankhaft gesteigerten Reizung der bekannten Wärmecentren, worlurch der motorisch-trophische Apparat der Körpermuskeln und der Gefässmuskeln zu erhöhter Wärmeproduction, gesteigertem Stoffverbrauch und Veränderung in der Wärmeabgabe angeregt wird. -

Die Mehrzahl der bisherigen Untersuchungen sind am Streifenhügel des Kaninchens angestellt. Ich selbst habe, um die Beziehungen des Nucleus caudatus zu Temperatursteigerungen zu prüfen, eine Reihe von Versuchen an $\mathrm{Hunden}$ angestellt. Ich habe, wie die meisten der bisherigen Untersucher, die Methode des Einstiches in den Nucleus caudatus gewählt (den sogen. Wärmestich). - Der Weg, auf dem ich den Nucleus caudatus dabei zu treffen suchte, ist derselbe, wie ich ihn für die Exstirpation des Nucleus caudatus angegeben habe, nämlich der von vorne her, durch das Stirnhirn hindurch. Es ist dies eine Methode, welche nicht nur relativ grosse Sicherbeit im Auffinden des Nucleus caudatus gestattet, sondern auch physiologisch anscheinend weniger wichtige Partien des Gehirns in Mitleidenschaft zieht. Die Operation wurde stets zweizeitig vorgenommen. Im Detail gestaltete sie sich folgendermaassen:

Der Hund wurde zuvor einige Tage beobachtet und Temperaturmessungen im Rectum an ihm vorgenommen. Es wurde sodann bei 
der ersten Operation in Chloroformäther- oder combinirter Morphinäthernarkose beiderseits die vordere und hintere Wand des Antrum frontale entfernt und das Stirnhirn freigelegt, die Dura aber nicht eröffnet. Nachher blieb das Thier 3-4 Tage lang in genauer Beobachtung: es wurden regelmässige Temperaturmessungen vorgenommen. Nach Ablauf dieser Zeit wurde in leichter Chloroformäthernarkose möglichst rasch die Hautwunde geöffnet, die Dura auf einer Seite gespalten und ein dünner Troikart in den Nucieus caudatus eingestochen. Gleich hernach wurde die Wunde durch Naht geschlossen. Unmittelbar darauf wurde ein Maximalthermometer in den Mastdarm des Hundes eingelegt und der Temperaturverlauf durch häufigeres Revidiren des Thermometerstandes constatirt. Das Thier konnte meist nach weiteren zwei Tagen zur Wiederholung des Wärmestiches auf der anderen Seite verwendet werden. Ich hebe ausdrücklich hervor, dass alle Thiere, über welche ich hier berichte, kurze Zeit (10 Minuten bis $1 / 2$ Stunde) nach dem Einstich, zu welchem meist nicht mehr als $1 / 2$ Stunde Zeit gebraucht wurde, das ihnen dargereichte Fleisch frassen, dass sie weiterhin keinerlei entzündliche Reactionserscheinungen an der Wunde zeigten, dass das Allgemeinbefinden während der ganzen Zeit der Beobachtung ein tadelloses war.

Ich lasse nunmehr die kurzen Protokollanszüge der einzelnen Versuche folgen.

\section{Versuch $\mathbf{T}_{1}$.}

An dem der Operation vorhergehendem Tage betrug die Mastdarmtemperatur $38,6^{\circ}$ C. (Vomittags), $: 8,4^{\circ}$ (Nachmittags). Am Tage der 1 . Operation, numittelbar vor dieser, wnrde 39,2 gemessen.

1. Operation, am 27. Februar 1902. Fntfernung des Stirnbeines, Freilegung des Stirnhirnes. In den dieser Operation folgenden vier Tagen überstieg die Temperatur kein einziges Mal den höchsten vor der Operation testgestellten Grad $\left(39,2^{\circ}\right)$.

2. Operation, am 3. März 1902. Wärmestich auf der linken Seite. Unmittelbar zuvor hatte die Temperatur $39,0^{\circ}$ betragen; sie stieg während der folgenden 48 Stunden niomals über $39,1^{\circ}$.

3. Operation, am 6. Mйirz 1902. Wärmestich auf der rechten Seite. Die Temperatur, welche unmittelbar vor dieser Operation $39,8^{\circ}$ betragen hatte, sticg in der 5 . Stunde nachher auf $40,2^{\circ}$. Von da an blieb sie in den folgenden Tagen $\mathrm{z}$ wischen $38,9^{\circ}-39,6^{\circ}$.

Die Section ergab, dass der anf der linken Seite vorgenommene Einstich, in dessen Gefolge kein Temperaturanstieg eingetreten war, das Mark des Stirnhirns durchsetzt, zum Centrum des Nucleus caudatus gelangt und sich innerhalb desselben weiter nach hinten und basalwärts fortsetzt. Kein Theil der Umgebung des Schweifkernes ist verletzt. 
Der Einstich auf der rechten Seite, nach welchem die Temperatur (5 Stunden später) eine Steigerung um $0,4^{0}$ über den höchsten Werth, den sie jemals vorher oder nachher zeigte, erreicht hatte, geht an der lateralen Seite des Stirnhirnmarkes zur dorsalsten und lateralsten Ecke des Schweifkernkopfes. Der Stich zieht sich dann längs des dorsalen Randes des Nucleus caudatus bis in den Schweif desselben hinein. Er trifft also von der Substanz des Nucleus caudatus nur den lateralen dorsalen Randtheil.

\section{Versuch $\mathbf{T}_{2}$.}

An den Tagen vor der ersten Operation betrug die Temperatur $39,5^{\circ}$.

1. Operation, am 13. März 1902. Freilegung des Stimhirnes. Die Temperatur stieg in den folgenden 72 Stunden niemals über $39,9^{\circ}$.

2. Operation, am 17. März 1902. Wärmestich links. Die Temperatur, welche unmittelbar vor dem Einstich 40,8 betragen hatte, stieg im Anschluss an die Operation auf $41^{\circ}$. Vom nächsten Tage an blieb sie unter diesem Werth.

3. Operation, am 19. März 1902. Wärmestich rechts. Die Temperatur, welche vor der Operation $39,5^{\circ}$ betragen hatte, stieg im Laufe der nächsten Stunden und erreichte den höchsten Werth bei $41,0^{\circ}$. Hernach fiel sie in den nächsten Tagen auf den ziemlich constanten Werth von $39,5^{\circ}$.

Die Section ergab: Der Stich auf der linken Seite, in dessen Gefolge die Temperatur eine Steigerung von $40,8^{\circ}$ auf $41^{\circ}$ gezeigt hatte, geht durch das Mark des Stirnhirnes in den Balken, unmittelbar über dem Nucleus caudatus, berührt denselben aber nicht. Der Einstich rechts, nach welchem ein Temperaturanstieg von $39,5^{\circ}$ auf $41^{\circ}$ eingetreten war, eine Temperatur, welche die höchste bei diesem Thier unter normalen Verhältnissen beobachtete um $0,2^{\circ}$ übertrifft, geht durch das Stirnhirn an die dorsale Seite des Nucleus caudatus und streift den freien dorsalen Rand desselben entsprechend der Mitte des Nucleus caudatus.

\section{Versuch $\mathbf{T}_{3}$.}

Die Temperatur an dem der Operation vorhergehenden Tage betrug $39,0^{\circ}$.

1. Operation am 26. März 1902. Freilegung des Stirnhirnes. Die höchste in den folgenden 2 Tagen erreichte Temperatur ist 39,10.

2. Operation am 28. März 1902. Wärmestich links. Die Temperatur, welche unmittelbar vor dieser Operation $39,3^{\circ}$ betragen hatte, steigt innerhalb der ersten 5 Stunden hernach auf $39,9^{\circ}$. Sie bleibt in den folgenden Tagen zwischen $39,1^{\circ}$ - $39,6^{\circ}$.

Bei der Section zeigte sich, dass in diesem Fall, wo die Temperatur in den dem Einstich folgenden Stunden $39,9^{\circ}$ erreichte, das ist $0,3^{\circ}$ mehr als die höchste bei diesem Thier jemals beobachtete Temperatur betrug, der Stich durch das linke Stirnhirn hindurchgegangen war; in diesem war es zu einer hämorrhagischen Erweichung im Mark gekommen. Weiter rückwärts gelangte der Einstich an die laterale Seite des Schweifkernes, zwischen diesen und die Kapsel. Er reichte bis in den hintersten Theil des Schweifkernes. Dieser selbst zeigte sich in ganzer Ausdehnung von zahlreichen kleinen Hämorrhagien durchsetzt. 


\section{Versuch $\mathbf{T}_{4}$.}

Die Temperatur vor der Operation betrug $39,6^{\circ}$.

1. Operation. Freilegung des Stirnhirnes. Die höchste nach dieser Operation erreichte Temperatur ist $39,7^{\circ}$.

2. Operation. Wärmestich links. Vorher war die Temperatur $39,0^{\circ}$. In den der Operation folgenden 8 Stunden steigt die Temperatur auf $40,2^{\circ}$. Sie fällt dann und erreicht in den nächsten Tagen höchstens $39,4^{\circ}$.

3. Operation. Wärmestich rechts. Die Temperatur, welche vor der Operation $39,4^{\circ}$ betragen, steigt im Laufe der nächsten 7 Stunden auf $41,3^{\circ}$. Sie fällt dann wieder und hält sich während der nächsten Tage zwischen 39,5 und $40^{\circ}$.

Die Section ergab: Der Einstich auf der linken Seite, nach welchem eine Temperatursteigerung aufgetreten war, welche die höchste bei dem betreffenden Thiere vorher beobachtete Temperatur um $0,5^{\circ}$ übertraf, ging durch das Mark des Stimhirnes in den Balken hinein, dorsomedial vom Nucleus caudatus. Er traf den Nucleus caudatus nux im vordersten Theile des Kopfes, dessen medialen Rand er streift. Weiter nach rückwärts reicht der Stich überhanpt nicht.

Rechte Seite: Hier war in den der Operation folgenden Stunden eine Temperatursteigerung auf $41,3^{\circ}$ eingetreten. Diese Temperatur übertrifft die vor der Operation um 1,9 $9^{\circ}$. Sie übertrifft die bei dem Thier unter normalen Verhältnissen beobachtete höchste Temperatur um 1,70. Bei der Section zeigte es sich, dass der Stich durch das Mark des Stirnhirnes hindurchging, hier eine grössere Blutung setzte; weiter rückwärts gelangte er an den dorsalen lateralen Wiukel des Schweifkernkopfes, durehsetzte diesen, kam dann in's Centrum des Nucleus caudatus und endete vor dem Schweif desselben. Er setzte keine Verletzung der Nachbarschaft des Nucleus caudatus.

Ueberblicke ich diese Versuche, so muss ich zunächst bemerken, dass die Zahl derselben noch eine recht geringe ist: im Ganzen sieben Einstiche an vier Thieren. In keinem Falle war durch die Voroperation irgend eine Temperatursteigerung veranlasst; die Temperatur, welche, wie ich nach einer grösseren Zahl von Messungen an normalen Hunden weiss, im Mastdarm zwischen $38,5^{\circ}-40^{\circ}$ schwankt, überstieg diesen Wert nach der Voroperation anch kein einziges Mal. Unter den sieben Fällen, in welchen der Finstich gemacht worden war, zeigte sich in einem Falle (Versuch $\mathrm{T}_{1}$ links) keinerlei Steigerung der Temperatur; in drei Fällen (Versuch $\mathrm{T}_{\mathbf{1}}$ rechts, $\mathrm{T}_{3}, \mathrm{~T}_{4}$ links) folgte dem Einstich in den nächsten Stunden eine geringe Temperatursteigerung, derart aber, dass keine übernormale Temperatur auftrat. In den restlichen drei Fällen (Versuch $\mathrm{T}_{2}$ links, $\mathrm{T}_{2}$ rechts, $\mathrm{T}_{4}$ links) trat dagegen übernormale Temperatur auf, und zwar in zwei Fällen Temperatursteigerung auf $41^{\circ}$, 
in einem Falle auf $41,3^{\circ}$. In dem einen dieser Fälle, wo die Temperatur $41^{\circ}$ erreichte (nämlich in Versuch $\mathrm{T}_{2}$ links), war sie allerdings nur um $0,2^{\circ}$ höher als die vorherige. In den beiden anderen dieser Fälle $\left(\mathrm{T}_{2}\right.$ rechts und $\mathrm{T}_{4}$ links) übertraf sie dagegen die vorhergehenden Temperaturen um ein Bedeutendes, das eine Mal um $1,5^{\circ}$, das zweite Mal um $1,9^{\circ}$.

In dem ersten dieser zwei Fälle ( $T_{2}$ rechts) ergab die Section, dass der Stich den dorsalen frejen Rand des Nucleus caudatus ungefähr entsprechend der Mitte desselben traf. In dem zweiten Falle $\left(\mathrm{T}_{4}\right.$ links) durchsetzte der Stich den Nucleus caudatus in seinem dorsalen Antheil, seiner ganzen Längenausdehnung nach. Es scheint also nach diesen wenigen Versuchen, dass in der That auch bei Hunden Einstich in den Nucleuscaudatus Temperatursteigerung in den ersten Stunden nach der Operation zur Folge hat. Diese Steigerung ging in einem Falle biszu $1,9^{\circ}$. - Sie scheint sich an ehesten einzustellen, wenn der dorsale mediale Antheil des Nucleus caudatus vom Stich getroffen ist. Anscheinend sind kleine Verletzungen wirksamer als umfangreichere Zerstörungen des nucleus caudatus. -

Die vorliegenden Untersuchungen wurden im physiologischen Institute des Herrn Geheimrathes Hermann Munk in Berlin begonnen und zum grössten Theil im Institute für allgemeine und experimentelle Pathologie des Herrn Professor Richard Paltauf in Wien durchgeführt.

Diesen Herren erlaube ich mir an dieser Stelle meinen ergebensten Dank auszusprechen.

Ueberdies bin ich für die Anregung zu dieser Arbeit Herrn Geheimrath Hermann Munk, für die Anleitung und ständige Förderung dabei Herrn Professor Biedl zu innigstem Danke verpflichtet.

In der Durchführung der Versuche wurde ich von Herrn Assistenten Dr. Rothberger in der liebenswürdigsten Weise unterstützt. 


\section{Literatur-Uebersieht.}

1) Aronsohn, Das Wesen des Fiebers. Deutsche medic. Wochenschr. 1902.

2) Bechterew, Petersburger medic. Wochenschr. 1881.

3) Bechterew u. Mislawsky, Ueber den Einfluss der Grosshirnrinde auf den Blutdruck und die Herzthätigkeit. Neurologisches Centralbl. 1886 Nr.9.

4) Bechterew, Untersuchungsergebnisse, betreffend die Erregbarkeit des hinteren Absehnittes des Stimlappens. Arch. f. Anat. u. Physiol. 1899 S. 503 .

5) Beyerman, over den invloed van het zenuwstelsel op de ademhaling. Dissert. Amsterdam 1900.

6) Bochefontaine, Etude experim. .... sur quelque fonctions de la vie organique. Arch. de physiol. normale et pathol. vol. 3 p. 2. 1876.

7) Christiani, Ein Athmungscentrum am Boden des 3. Ventrikels. Centralblatt f. d. medic. Wissenschaften. 1880.

8) Christiani, Zur Physiologie des Gehirns. Berlin 1885.

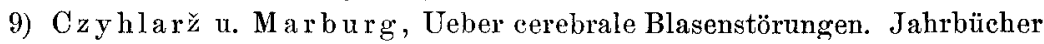
für Psychiatrie u. Neurologie. 1901.

10) Danilewsky, Experimenteller Beitrag zur Physiologie des Gehirns. Pflüger's Archiv 1875.

11) François-Franck, Leçons sur les fonctions motrices du cerveau. Paris 1887.

12) It 0 , Ueber den Ort der Wärmebildung nach Gehirnstich. Zeitschr. für Biologie Bd. 20 Heft 1. 1899. (Umfassende Literaturzusammenstellung.)

13) Landois, Lehrbuch der Plyysiologie.

14) Lépine, Compt. rend. de la Soc. de Biolog. 1875.

15) Lewandowski, Die Regulirung der Athmung. Arch. f. (Anatomie u.) Physiologic. 1896.

16) Lo Monaco, Sulla fisiologia del corpo calloso e sui mezzi d' induzione per lo studio dei gangli della base. Riv. di patol. nerv. e mental. 1897.

17) Marckwald, Die Bedeutung des Mittelhirns für die Athmung. Zeitschrift f. Biologie Bd. 26.1890.

18) Martin u. Booker, John Hopkins Univers., Studies from the biolog. laboratory. Baltimore 1879.

19) Martin u. Booker, Journ. of Physiol. tom. 1 p. 570.

20) Minor, Zur Frage über die Bedeutung des Corpus striatum. Dissert. Moskau 1882.

21) H. Munk, Ueber die Sinnessphären. Sitzungsber. d. königl. preuss. Akad. d. Wissensehaften zu Berlin. 1882.

22) H. Munk, Ueber die Ausdehnung der Sinnessphären in der Grosshirnrinde, 2. Mittheil. Ebendasclbst 1900.

23) Nothnagel, Experimentelle Untersuchungen über die Functionen des Gehirns. Virchow's Archiv Bd. 57 u. 58. 1873.

24) Preobraschensky, Ueber Athemcentren in der Hirnrinde. Wiener klin. Wochenschr. $1890 \mathrm{Nr} .41$ u. 43. 
508 Arthur Schüller: Reizversuche am Nucleus caudatus des Hundes.

25) Prus, Ueber die bei elektrischer Reizung des Corpus striatum und des Thalamus opticus auftretenden Erscheinungen. Wiener klin. Wochenschr. $1899 \mathrm{Nr} .48$.

26) Richet, Des circonvol. cérébral. Th. agrég. Paris 1878.

27) Schukowsky, Ueber den Einfluss der Hirnrinde und der subcerticalen Ganglien auf die Athmung. Neurolog. Centralbl. 1898 Nr. 3.

28) Spencer, The effect produced upon Respiration by farad. Excit. of the cerebrum in Monkey, Dog, Cat and Rabbit. Philos. transact. tom. 185 p. 609.1894 .

29) Spencer, Lectures on the centr. n. syst. Lancet 1895 .

30) Stricker, Untersuchungen über die Gefässnervencentren im Gehirn und Rückenmark. Medicin. Jahrbücher. 1886 .

31) Unverricht, Experimentelle Untersuchungen über den Mechanismus der Athembewegungen. Klin. Centralbl. 1888.

32) Wieting, Zur Physiologie der infracorticalen Ganglien. Inauguraldissert. Dorpat 1891.

39) Ziehen, Function der subcorticalen Ganglien. Archiv für Psychiatrie Bd. 20. 1889.

34) Ziehen, Physiologie der infracorticalen Ganglien. Archiv für Psych. Bd. 21. 1890. (Literaturübersicht der Reizversuche am Streifenhügel.) 